\title{
Mobile-assisted learning and higher-education ESP: English for physiotherapy
}

\author{
Lin Elinor Pettersson \\ Department of English, French and German, University of Málaga \\ pettersson@uma.es
}

\begin{abstract}
Lin Pettersson. Mobile-Assisted Learning and Higher-Education ESP: English for Physiotherapy. The Poznań Society for the Advancement of Arts and Sciences, PL ISSN 0079-4740, pp. 81-94

The popularity of Mobile-assisted language learning has increased significantly in recent years, and language teachers are still exploring different ways of introducing new technology into the language classroom. Up to the moment, this has mainly been achieved through the use of mobile language-learning applications (Grimshaw et al. 2017). We wanted to push the use of applications in the classroom of English for Specific Purposes further by introducing a human anatomy application in the context of English for physiotherapy in higher education. We believe that the use of an application for a specific area provides the opportunity to enrich the learning experience and take language-learning outcomes to a different level as students are granted a unique occasion of applying knowledge acquired in a specialist area in the language classroom. An intervention proposal was designed for the subject English for physiotherapy (University of Málaga, Spain) bringing together mobile-assisted language learning with a task-based approach to suit constructivist learning processes and accommodate different learning styles and rhythms. We followed five guiding principles on mobile-assisted language learning (Stockwell \& Hubbard 2013) to design three tasks for autonomous and collaborative learning using the application 3D4 Medical Essential Anatomy. A preliminary survey was carried out using Lime Survey to measure under-graduate physiotherapy students' attitudes towards the use of a human anatomy application to learn English and to predict possible challenges (language, technology, cost or storage capacity). The results support our belief as the obtained data indicates that students perceived the use of a human anatomy application as an interdisciplinary tool for both educational and professional purposes and were willing to purchase an application to learn English for physiotherapy.
\end{abstract}

Keywords: MALL, ESP, English for physiotherapy, 3D applications, task-based language learning

\section{Introduction}

The pedagogical implications of Mobile-Assisted Language Learning (henceforth MALL) are rich and varied, and this innovative method has been defined as "mobile technologies in language learning, especially in situations where device portability offers specific advantages" (Kukulska-Hulme 2013: 3701). MALL includes different mobile devices, and we are particularly interested in the use of material with touchscreen and 
mobile operating systems as smartphones and tablets. These are becoming increasingly popular in Foreign Language (henceforth FL) teaching and as they are perceived as highly innovative by teachers and as very attractive by learners.

One of the main advantages is that MALL places the learner in focus, especially through the use of different applications (henceforth apps). Several studies have demonstrated that the use of apps in FL favours independent learning and boosts learner autonomy. Michael Thomas (2012) has highlighted that the use of Web 2.0 applications not only provides authentic learning environments and stimulates learners' engagement in real-world activities, but also fosters learner autonomy; particularly through the use of interactive tasks. Moreover, Jason Byrne (2016: 74) draws attention to how recent research into MALL has shown that "mobile-learning apps offe[r] excellent opportunities for personal learner-centred study" (see Kim \& Kwon 2012; Ahmad \& Farrukh 2015). In fact, teachers and learners have perceived language-learning apps as more suitable for individual and independent studies than for interpersonal and communicative learning (Byrne 2016: 80-81). However, we strongly believe that apps have great potential for both intra- and inter-personal language learning.

We are particularly interested in the pedagogical implications of using apps in the context of English for Specific Purposes (ESP). This requires a careful selection of a suitable app, as well as meticulous planning of tasks and activities that respond to the specific needs of the learners. Jennica Grimshaw et al. (2017: 137) point out that MALL methodology in foreign language learning has mainly been based on the use of language learning applications, or on allowing students to resort to mobile devices to look up words and definitions in on-line dictionaries, search the web, take notes or play language games. A similar approach has been adapted in higher education ESP. For example, attempts have been made to introduce MALL methodology in ESP in technical and engineering education at the University of Hradec Kralove (Czech Republic). Teachers used an app called 'application for practising vocabulary' (or AVP), which included translation of words, glossaries and lexical exercises based on error analysis, gap-filling and multiple-choice activities (Simonova 2016: 35). We want to invite teachers of ESP to move beyond language-learning apps and explore the richness and abundance of high-quality apps available in English within different disciplines.

Higher-education ESP ideally responds to the specific language demands and learning processes of students in different disciplinary areas. A major challenge for teachers is that they are not necessarily experts in the area they teach English. In our case, it is most likely that the English teacher - in contrast to the students - has no formal training in human anatomy. While students have performed autopsies to study human anatomy, English teachers have not. Thanks to the 3D anatomical app, language teachers can now get familiar with human anatomy in a way that was previously impossible. What is more, the use of a 3D anatomical app offers learners the possibility to unite advanced theoretical knowledge and practical skills in physiotherapy with ESP.

We propose combining MALL methodology with a task-based approach when using specialised apps available in English. Thomas (2012) has noticed that the development of Task-Based Language Teaching (TBLT) and Computer-Assisted Language Learning (CALL) has been continuous and parallel since the 1990s. Accordingly, TBLT has situ- 
ated itself as one of the most important popular methodologies within FL learning while the development of CALL, and subsequently MALL, has been exceptional. One point in common is that the focus is on the learner. ESP scholars have similarly paid heed to the importance of the learning process. In fact, Tom Hutchinson \& Allan Waters have validated the task-based approach as the most efficient learning method in ESP claiming the following: "a truly valid approach to ESP must be based on an understanding of the process of language learning" (1987: 14; original emphasis). We have created a Venn diagram (Figure 1) to illustrate the overlap between MALL, TBLT and ESP.

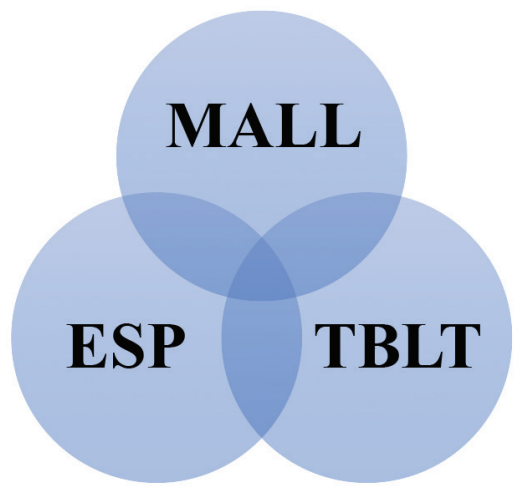

Figure 1. The shaded area demonstrates the overlap of MALL, TBLT and ESP

Taking this as a starting point, we encourage ESP teachers to select an appropriate app for the discipline, and then design suitable tasks and activities according to the established learning objectives. Given that we will pay heed to the process of learning rather than teaching, we will use the term TBLL to refer to task-based language learning from this point onwards. The following intervention proposal has been developed in the context of English for physiotherapy.

\section{Intervention proposal}

Under-graduate physiotherapy students are taught how to diagnose, evaluate and treat musculoskeletal short-term and long-term conditions and injuries. If exploring musculoskeletal structures by palpitation is one of the basic skills of the profession (Noguera et al. 2013: 96), knowledge of human anatomy is another key skill to assess and treat patients. At the University of Málaga (Spain), students of physiotherapy are taught human anatomy at the Faculty of Medicine during the first year. The subject combines theoretical seminars on human anatomy, where they study 2D images or cross-sections images acquired in CT scans, and practical sessions performing autopsies. During the second year they learn how to use manual therapy to explore and treat living patients in the teaching laboratory at the Faculty of Health Sciences. Students apply these skills with 
real patients during their clinical practice when they rotate different workplaces (hospitals, private clinics, health centres etc.) in the third and fourth years.

Students are also expected to learn English for physiotherapy to be able to perform their future profession in English as well as access academic and scientific texts in English. The subject 'English for physiotherapy' is obligatory and taught in the third year. Students are recommended to have a B1 level of English to be able to follow the course and the syllabus covers the following five parts:

- Introduction to English for Specific Purposes: English for physiotherapy.

- The musculoskeletal system in English.

- Basic concepts and terminology related to physiotherapy.

- English for clinical practice.

- Internationalization.

Our intervention proposal covers 'the musculoskeletal system in English', 'basic concepts and terminology related to physiotherapy' and 'English for clinical practice'.

As we aim to place the learner in focus, it is essential to take into account how students of physiotherapy learn, and if they have a dominant learning style. Milanese et al. (2013) carried out a study of physiotherapy students' preferred learning-styles in the context of clinical education. It was found that a constructivist learning style was the preferred one as the following characteristics were identified (Milanese et al. 2013: 148; adapted from Kolb 2005):

- Best at finding practical uses for ideas and theories.

- Have the ability to solve problems and make decisions based on finding solutions to questions and problems.

- Prefer to deal with technical tasks and problems rather than with social issues and interpersonal issues.

- These learning skills are important for effectiveness in specialist and technology careers.

- In formal learning situations, prefer to experiment with new ideas, simulations, laboratory assignments, and practical applications.

If students of physiotherapy prefer a constructivist learning style for clinical practice, then how can we adopt a constructivist learning approach in English for physiotherapy? Mobile-assisted language learning is not only an innovative method, but also highly motivating and stimulating for students. As Thomas argued, "technology can promote learner agency in language learning contexts, corroborating constructivist goals and marginalizing the notion that learners are merely empty vessels to be filled with knowledge poured into them by more knowledgeable instructors" (2012: 316). We propose the implementation of an interactive 3D anatomical app into the English classroom as we consider it to be an ideal learning tool for students of physiotherapy. Moreover, it is suitable for teachers who wish to adopt a constructivist approach for the following reasons: a) the use of a 3D app is innovative and attractive for students; b) it is interactive and dynamic; c) it fosters autonomous learning and is appropriate for both intra- and inter-personal learning; d) it is suitable for different learning rhythms and learning styles.

Previous attempts to introduce 3D anatomical apps into the teaching of physiotherapy have been made. At the University of Jaén (Spain), Nogera et al. have carried out an 
experimental study of how to incorporate 3D medical imaging into the classroom through mobile-assisted learning. The scholars identified four major advantages of 3D medical imaging as a learning tool for students of physiotherapy: (1) a directly recognizable visualization of anatomical structures; (2) reduction of the cognitive load as students do not need to construct their own mental visualization model; (3) infinite anatomical perspectives and the ability to interactively move the model for additional depth cues; and (4) the ability to include 3D models acquired from living human imagining datasets, eliminating the potential inaccuracies of stylized 2D drawings (2013: 96).

Since 3D anatomy apps present many affordances for students of physiotherapy, then they can also serve as a language-learning tool in English for physiotherapy.

Our intervention project has been planned for the subject 'English for physiotherapy', and our target group is a class consisting of 60 students who meet three hours weekly divided in the following way: two hours of theoretical sessions and one-hour session taken in three groups (20 students per group). The whole class meets in an ordinary classroom while the groups are taught in the teaching laboratory available at the Faculty of Health Sciences. All classrooms have free Wi-Fi access, and the teaching laboratory is equipped with 10 hydraulic treatment tables.

We aim to enhance the learner outcome in English for physiotherapy by introducing a 3D anatomical application in English. This way we hope to foster a motivational learning environment that incorporates authentic and innovative material using a task-based method that stimulates both inter- and intra-personal learning through interactive tasks and activities. Our principal aim is twofold: on the one hand, students will learn the musculoskeletal system and specific terminology for physiotherapy in English and on the other hand they will be able to use it in clinical practice. We believe that apps possess great potential not only for autonomous and independent learning, but for interpersonal learning as well.

Our method is guided by five principles for MALL established by Glenn Stockwell \& Philip Hubbard (2013: 9-10):

- Acknowledge and plan for accommodating language learner differences.

- Keep mobile language learning activities and tasks short and succinct when possible. As a corollary, divide longer tasks or activities into smaller, coherent chunks.

- Let the language learning task fit the technology and environment, and let the technology and environment fit the task.

- Some, possibly most, learners will need guidance and training to effectively use mobile devices for language learning.

- Recognize and accommodate multiple stakeholders. In the language classroom setting, adequate preparation and motivational support for teachers as well as learners must be provided.

We build our methodology on a task-based approach and have developed three tasks using a 3D human anatomy app:

Task 1: Students will create a musculoskeletal glossary during the whole semester. This task focuses on vocabulary and autonomous learning.

Task 2: Students will perform a detailed study of the musculoskeletal system of a specific area in the teaching laboratory. Two or more learners are involved in this task to 
put vocabulary into practice and develop interactional skills between physiotherapists and patients.

Task 3: A problem-solving task has been designed to expand vocabulary and to develop English communicative strategies and interactional skills between physiotherapists. Thus, at least two learners are involved in this task.

We have selected the app 3D4 Medical Essential Anatomy for our intervention proposal. It has been developed by 3D4 Medical - a 3D technology platform, which offers a series of highly specialised applications on the human body. Different versions are available for Android and Apple devices and students can use either 3D4 Medical Essential Anatomy 3 or 5. Depending on which version the students purchase, 3D4 Medical Essential Anatomy is available from 9.99 Euros.

The use of a 3D application on human anatomy enables both teachers and students to visualize the musculoskeletal system in ways they have not experienced before. Hayo Reinders \& Philip Hubbard highlight that a major challenge for teachers who wish to incorporate technology into the classroom is that "[m]aterials frequently lack the necessary support structures, such as clear instructions or even answer keys, and do not explicitly encourage students to reflect on the learning process" (2012: 335). This is not the case with 3D4 Medical Essential Anatomy; this application offers a comprehensive and user-friendly tool to explore human anatomy. Instruction videos on how to use the app and make the most of the different functions and options are available on the online platform. Several functions facilitate a detailed examination of the 3D anatomy model (male and female), and users can:

- listen to pronunciation,

- read information,

- take notes,

- add pins,

- visualize specific areas in several ways by activating options as 'isolate', 'hide', 'fade', 'fade others', 'hide others',

- rotate model to obtain alternative angles,

- zoom in and zoom out,

- activate multiple-selection mode,

- choose 'black', 'white' or 'vignetting' (i.e. shaded) background,

- test progress in different quizzes with drag- and drop or multiple-choice questions with a key.

The app has a pre-installed tutorial to familiarize users with the different options and functions the app offers. However, the teacher will complement this with a tutorial in class in case students find it difficult to understand instructions in English.

\subsection{Task-based language learning in ESP: English for physiotherapy}

The first task consists in elaborating an individual glossary of the musculoskeletal system in English using the app. This favours both interdisciplinary and autonomous learning. Our task differs from the traditional use of bilingual dictionaries as instead of 
looking up words, the students can explore the human body and visualize its muscles and bones, as well as rotate the part or zoom in and out to obtain multiple perspectives. As students prepare an English glossary of the musculoskeletal system they use previous knowledge in human anatomy. Additionally, different options available in the app offer multiple ways of acquiring new vocabulary: users can read definitions, learn about characteristics, listen to pronunciation and add their own notes. In doing so, we hope to achieve a "language learning that go[es] beyond simple replication of paper-based or even computer-based learning materials" (Stockwell \& Hubbard 2013: 4). We recommend preparing the first part of the glossary in class so the teacher can monitor the initial use of the app and help with possible language difficulties. Students will complete the rest of the glossary at home. To divide a lager project of learning the musculoskeletal system into a smaller and comprehensible task, the teacher will set several deadlines to complete different areas throughout the semester.

The second task we propose is a case study of a specific area of the musculoskeletal system. This could be any area of the body, but we recommend concentrating on those related to injuries commonly treated by physiotherapists. As shoulder injuries are among the most common conditions in physiotherapy, we have decided to design a task that focuses on the shoulder rotator cuff. Nevertheless, our task can be expanded or adapted to other parts of the body.

Students will study and examine the muscles, tissue and bones involved in an interactive and communicative way to develop both intra- and interpersonal learning. Before class, students will be asked to explore the shoulder area and to identify and study the parts that build the rotator cuff. In the process, they will both learn human anatomy in English and develop their technical literacy using the 3D anatomical app. The task focuses on the infraspinatus muscle taking the following information (provided by the app) as a starting point:

- Infraspinatus (Musculus infraspinatus).

- Origin: Infraspinous fossa of the scapula.

- Insertion: Greater tubercle of the humerus.

- Action: External rotation of the arm.

- Nerve supply: Supracapular nerve (C4-C6).

- Arterial supply: Circumplex scapular artery; suprascapular artery.

- Clinical: The tendon of this muscle is one of four that forms the rotator cuff of the shoulder joint. Repeated abduction and flexion can lead to wear and tear, causing injury of rupture of this cuff. Lesions of the suprascapular nerve can result in weak shoulder rotation and posterolateral shoulder pain.

During our face-to-face session, students meet in groups in the teaching laboratory where they will work in pairs. One stretcher is available for every couple and all students will bring their mobile devices to class. This task is suitable for different learning styles and learning rhythms as students can visualize the area in an interactive way according to their individual needs and get accustomed to using the app at their own pace. Thomas has argued that "technology can provide opportunities to transcend the limitations of the traditional classroom context. Online materials and applications can significantly enhance the types, authenticity and range of tasks that learners engage in" (2012: 317). By 


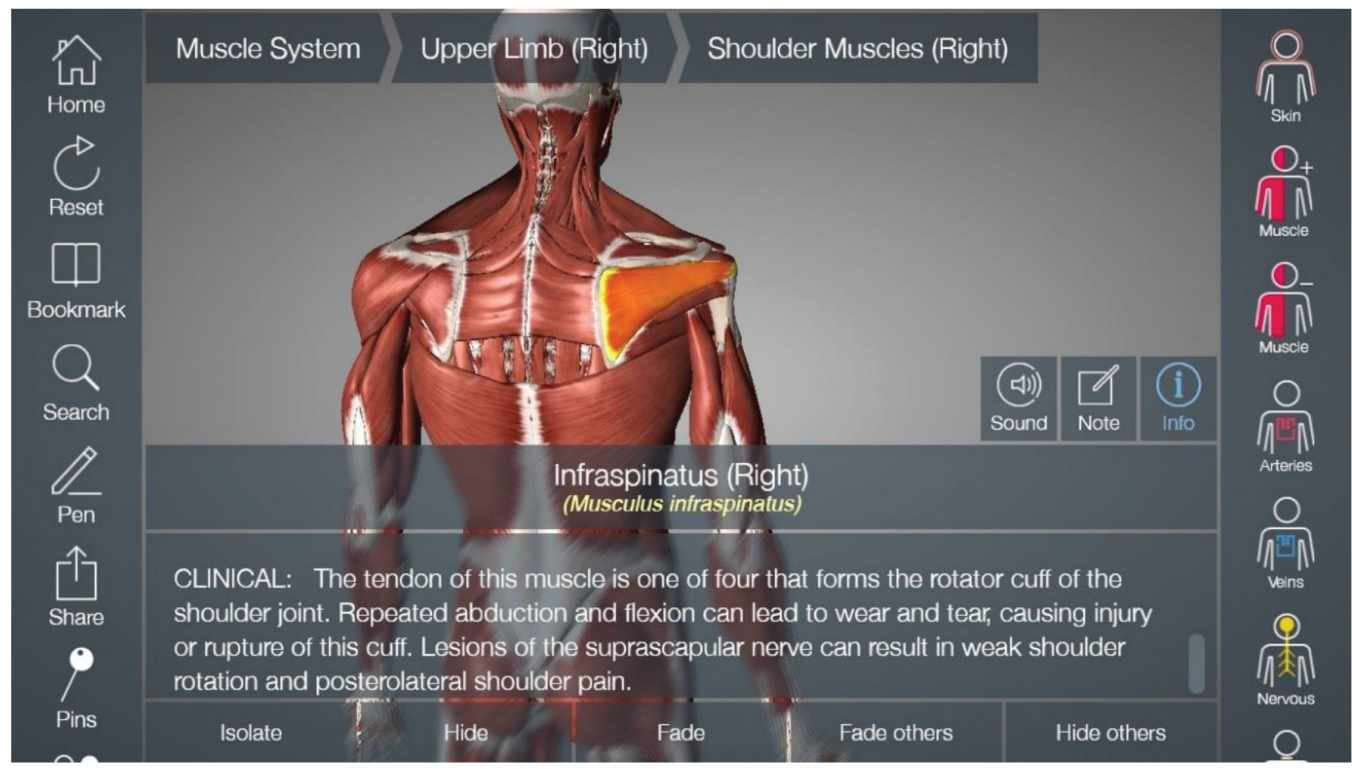

Figure 2. Screenshot presenting an overview of available options and functions to study the infraspinatus muscle. 3D4 Medical Essential Anatomy 5

moving the English class into the physiotherapy teaching laboratory we hope to provide an authentic learning environment as well as foster learners' engagement in real-world activities by incorporating a 3D anatomical app.

Students will first visualize the muscles and bones of an affected area using the app. By using a multiple selection mode, they can single out the infraspinatus muscle and scapula bone. Thanks to the options 'fade others' and 'isolate', the learners can study a specific area of interest in detail (see Figures 3 and 4). Once students have visualized the area in their mobile devices, they will engage in physiotherapist - patient role play and the physiotherapist proceeds to exploring the muscle on the patient's body. As the infraspinatus muscle is situated on the posterior aspect of the scapula bone, students will locate the muscle on the partner's body by first touching the scapular spine and then tracing the thick triangular muscle with his or her fingers. This part of the task provides the opportunity for learners to develop communicative skills in English for physiotherapy by practicing physiotherapist - patient dialogue in an authentic milieu.

In order to understand the connection between the infraspinatus muscle and the nervous system students will be asked to complete two steps of identification: first, they will isolate the infraspinatus muscle and the nerves that are directly connected to the muscle using 'multiple selection mode'. Once the specific parts have been selected, students will activate the 'fade others' option to study the relationship between muscle and nerves in detail (see Figure 5). This way, students will get insight into the possible areas affected and will be invited to discuss the probable peripheral signs, symptoms and types of pain a patient may have. 


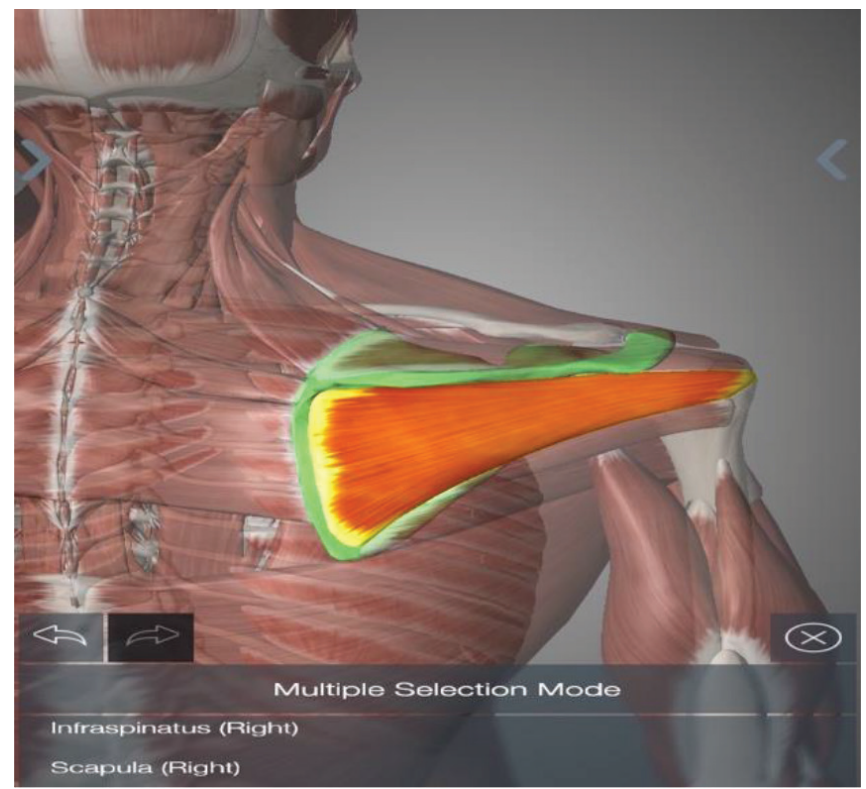

Figure 3. Screenshot of infraspinatus muscle and scapula bone (multiple selection mode; fade others). 3D4 Medical Essential Anatomy 5

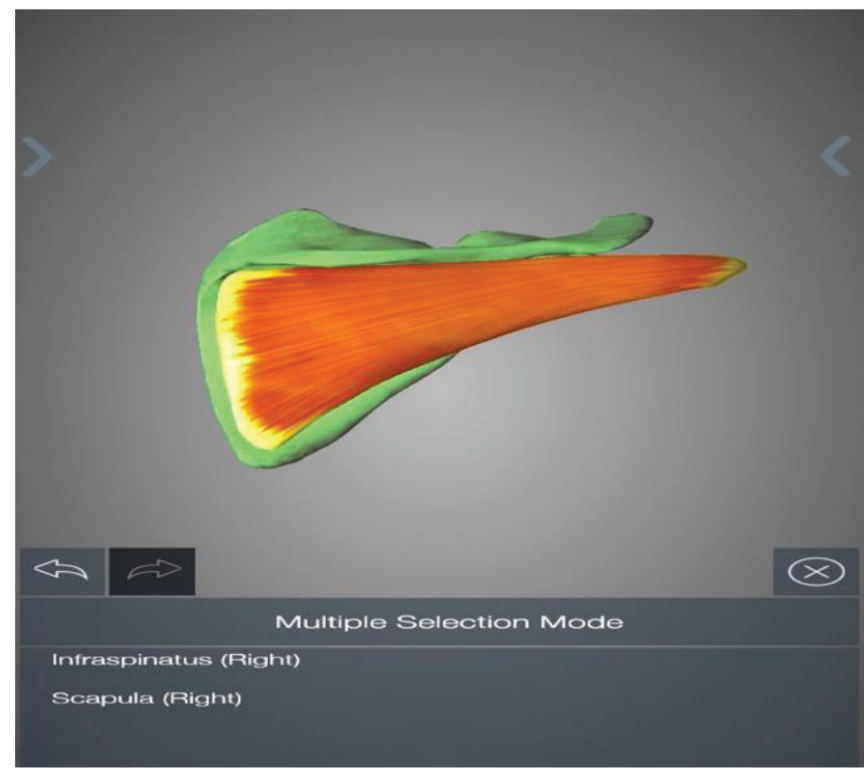

Figure 4. Screenshot of infraspinatus muscle and scapula bone (multiple selection mode; hide others). 3D4 Medical Essential Anatomy 5 


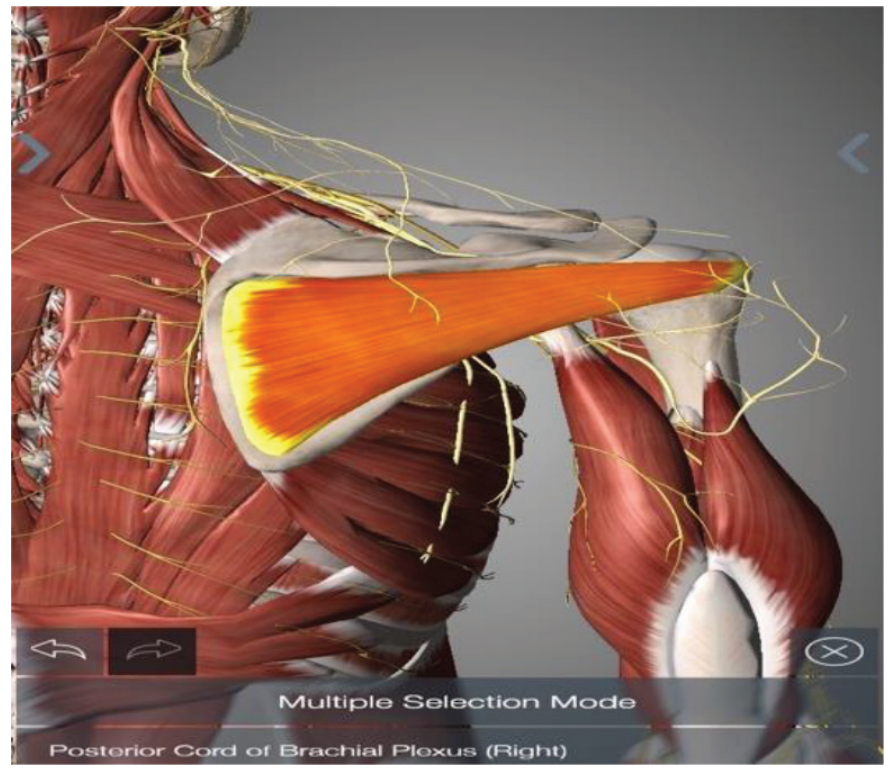

Figure 5. Screenshot of infraspinatus muscle and scapula bone, with the nervous system visible (multiple selection mode; fade others). 3D4 Medical Essential Anatomy 5

The third task is a problem-solving activity in which the students will engage in a physiotherapist - patient role play following the procedure of clinical practice. In this task, students will practice diagnosis, evaluation and treatment of their patients in English using the app as a supportive tool. Reinders \& Hubbard have argued that "pedagogical materials can be easily created, shared and updated, with learners potentially contributing to this process [...]. It gives learners [the] control they lack in more traditional environments" (2012: 332). As in the previous tasks, the focus will remain on the learners and will additionally rely on their previous experience from physiotherapist training and clinical practice.

Building on the previous task on the anatomical features of the rotator cuff, students will proceed with completing a problem-solving task in the teaching laboratory analysing infraspinatus injuries. These are commonly associated with repetitive over-head movements or caused by maintaining the arm in an elevated or extended position for longer periods:

- Common sports injuries: muscle tear of the infraspinatus (related to sports such as volleyball, handball or basketball among others).

- Possible occupational injuries: constant or bad postures of the arm in professions where people work behind a desk, for example mouse motion at the computer, may cause micro tears in the infraspinatus muscle, or in catering and restaurant service staff who carry the tray in an elevated position (the left shoulder is more commonly affected). 
- Injuries caused by poor or repeated postures: maintaining the arm in an elevated position for a longer period, as for example driving with one arm on the upper hand of the wheel.

Each student will be assigned a patient role and subsequently the physiotherapist identifies the problem, establishes a diagnosis and decides on an appropriate treatment. During this inter-communicative task, students practice English for physiotherapy by a) asking and answering questions; b) describing signs and symptoms; c) giving instructions to patients; and d) making suggestions and giving advice.

The 3D app serves as a useful tool to solve the problem and treat the patient. José M. Noguera et al. highlight that "the possibility to examine a 3D reconstruction of the musculoskeletal architecture [...] would lead to a more precise evaluation of the pathological situation of the patient and easier determination of hand locations during the practice class of manual therapy" (2013: 106). Finally, as the app offers the possibility of placing pins and adding notes, students would use the app to write down their solution to the problem.

\section{Preliminary study}

\subsection{Method}

We wanted to find out about our students' attitudes towards the use of MALL in 'English for physiotherapy', and anticipate whether possible drawbacks, such as cost, technological literacy or storage capacity (Reinders \& Hubbard 2012) would pose significant challenges for our undergraduate students. We used Lime Survey to create a mixeditem questionnaire consisting of five-point Likert scales and multiple-choice and yes/no questions. 15 students participated in the on-line questionnaire, which took three minutes to complete.

\subsection{Results}

The general outcome showed that the majority felt positive towards using a human anatomy app and stated that they would use it to study English for physiotherapy (20\%), to study English for physiotherapy and other subjects in the degree (53.33\%), in professional contexts $(40 \%)$, to study and in professional contexts $(73.33 \%)$. The answers indicate that students perceive the app as an interdisciplinary tool that can be used both in educational contexts and for professional purposes.

As one of the main challenges is the cost, we asked students what amount they considered to be feasible to pay. We also considered the possibilities of using only an app, only a text book or a combination of both. When students were asked whether they would buy an app to replace a text book, 12 students (80\%) would agree to do so, whereas 3 students (20\%) would prefer a book to an app. We believe that this result is related to the question regarding the amount they would be willing to pay for an app: 3 students 
(20\%) stated that they would not pay for an app. Others answered that they would pay $10-15$ euros $(46.67 \%)$ and very few $(13.33 \%)$ would pay $15-20$ euros or an amount higher than the cost of a text book of 25 euros (13.33\%). Yet, if an app replaces a text book, more students would be willing to pay 25 euros (33.33\%). This means that students are willing to purchase an app if the cost is not too excessive. Yet, if it amounts to an additional expense to a text book, they are less inclined to pay for it.

When asked about possible inconveniences of using an app in English for physiotherapy, surprisingly few students considered that the English language would be an obstacle. Only 2 students found it difficult to use an app in English, while none of the participants would have difficulties using technology. However, many students felt worried about not understanding the instructions in English (33.33\%). The main problem that students predicted was the space the app would occupy on their mobile devices (46.67\%). 2 students $(13.33 \%)$ added that they could not find any inconveniences at all.

Students were asked about the possible advantages of using a 3D anatomical app taking into account language learning as well as educational and professional aspects. This was a multiple-choice question and students believed that the app offered the following benefits: to improve level of English (60\%), for interdisciplinary learning (66.67\%) and to synthesize theory with practice $(73.33 \%)$. One student added that it could also be a useful tool to show patients their affected body parts in their future professional careers $(6.67 \%)$.

Finally, when students were asked if they would like to learn human anatomy in English using a 3D anatomical app students' responses were highly positive: $93.33 \%$ confirmed that they would like to learn human anatomy using an app.

\section{Conclusion}

We have proposed to implement a 3D anatomical app in the ESP classroom in the subject English for physiotherapy placing the learner in focus. In doing so, we have brought together MALL, TBLL and ESP (see our Venn diagram in Figure 6) to design tasks that accommodate different learning styles and rhythms, and that are interdisciplinary and highly motivational for students.

Our intervention proposal is based on previous research into MALL and we have also paid heed to previous attempts at introducing 3D anatomical apps in physiotherapy. We have designed three different tasks for the app 3D4 Medical Essential Anatomy following five MALL principles established by Stockwell \& Hubbard (2013: 340) in an attempt to meet "the need to begin by identifying efficient and effective techniques and procedures for using language materials or engaging in language learning tasks and activities mediated by technology". We strongly believe in the language-learning potential of using a highly developed app in specific discipline for ESP rather than using a language-learning app.

The benefits of using a highly developed and specialized app as 3D4 Medical Essential Anatomy clearly outweigh the challenges, and our findings demonstrate that students are initially aware of the interdisciplinary potential of using a 3D anatomical app as a tool 


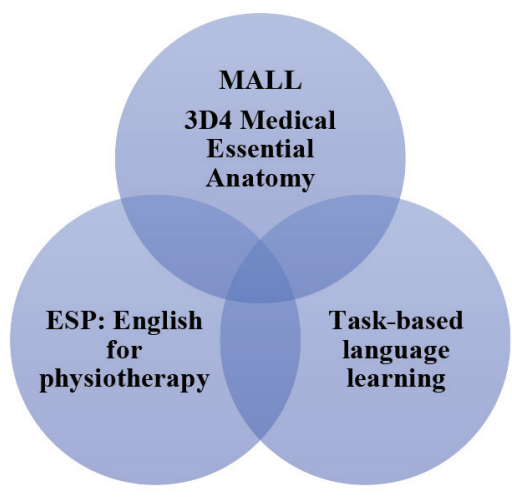

Figure 6. The shaded area demonstrates the overlap of MALL, TBLL and ESP

both in educational and professional contexts. The data collected in the questionnaire shows that students are very positive towards the use of a specialized app in the classroom and also that they would for pay for an app as long as it is not too costly. As the majority of students admit that they would pay for the app 3D4 Medical Essential Anatomy, and nearly all students would like to study human anatomy in English using the app, the next step in our research is to carry out an experimental study of our intervention proposal during the academic year 2018/19.

\begin{abstract}
Abbreviations
3D - three dimensions; App - applications; Computer-assisted language learning - CALL; English for specific purposes - ESP; Foreign language - FL; Mobile-assisted language learning - MALL; task-based language teaching - TBLT; task-based language learning - TBLL.
\end{abstract}

\title{
References
}

3D4 Medical Essential Anatomy 5. 2018. https://3d4medical.com/apps/essential-anatomy-5. (Accessed 201802-13.)

Ahmad, Anmol \& Farruk, Fizza. 2015. Significance of social applications on a mobile phone for English task-based language learning. Teaching English with Technology 15(2). 94-105.

Byrne, Jason. 2016. Same time same place: Do MALL classrooms exist? Teaching English with Technology 3(16). 74-84. http://www.tewjornal.org. (Accessed 2018-03-05.)

Grimshaw, Jennica \& Cardoso, Walcir \& Collins, Laura. 2017. Teacher perspectives on the integration of mobile-assisted language learning. In Borthwick, Kate \& Bradley, Linda \& Thouësny, Sylvie (eds.), CALL in a climate of change: Adapting to turbulent global conditions. Research-publishing.net, 135-139. http//:doi.org/10.14705/rpnet.2017.eurocall2017.702. (Accessed 2018-02-23.)

Hutchinson, Tom \& Waters, Allan. 1987. English for Specific Purposes: A learning-centred approach. Cambridge: Cambridge UP.

Kim, Heyoung \& Kwon, Yeonhee. 2012. Exploring smartphone applications for effective mobile-assisted language learning. Multimedia-Assisted Language Learning 15(1). 31-57. 
Kukulska-Hulme, Agnes. 2013. Mobile-assisted language learning. In Chapelle, Carol (ed.), The encyclopedia of applied linguistics, 3701-3709. New York: Wiley.

Milanese, S. \& Gordon, S. \& Pellat, S. 2013. Profiling physiotherapy student preferred learning styles within a clinical education context. Physiotherapy 99. 146-152.

Noguera, José M. \& Jiménez, Juan J. \& Osuna-Pérez, M. Catalina. 2013. Development and evaluation of a 3D mobile application for learning manual therapy in the physiotherapy laboratory. Computers and Education 69. 96-108.

Reinders, Hayo \& Hubbard, Philips. 2012. CALL and learner autonomy: Affordances and constraints. In Reinders, Hayo \& Warschauer, Mark \& Thomas, Michael (eds.), Contemporary computer-assisted language learning, 328-342. London: Bloomsbury.

Thomas, Michael. 2012. Task-based language teaching and CALL. In Reinders, Hayo \& Warschauer, Mark \& Thomas, Michael (eds.), Contemporary computer-assisted language learning, 312-327. London: Bloomsbury.

Simonova, Ivana. 2016. Mobile devices in technical and engineering education with focus on ESP. iJIM 10(2). $33-40$.

Stockwell, Glenn \& Hubbard, Philip. 2013. Some emerging principles for mobile-assisted language learning. The International Research Foundation for English Language Education, 1-15. https://www.tirfonline.org/ english-in-the-workforce/mobile-assisted-language-learning/some-emerging-principles-for-mobile-assisted-language-learning. (Accessed 2018-03-05.) 